\title{
Information metrics for improved traffic model fidelity through sensitivity analysis and data assimilation
}

\author{
A. Sopasakis * M. A. Katsoulakis ${ }^{\dagger}$
}

December 30, 2015

\begin{abstract}
We develop theoretical and computational tools which can appraise traffic flow models and optimize their performance against current time-series traffic data and prevailing conditions. The proposed methodology perturbs the parameter space and undertakes path-wise analysis of the resulting time series. Most importantly the approach is valid even under non-equilibrium conditions and is based on procuring path-space (timeseries) information. More generally we propose a mathematical methodology which quantifies traffic information loss.

In particular the method undertakes sensitivity analysis on available traffic data and optimizes the traffic flow model based on two information theoretic tools which we develop. One of them, the relative entropy rate, can adjust and optimize model parameter values in order to reduce the information loss. More precisely, we use the relative entropy rate as an information metric between time series data and parametrized stochastic dynamics describing a microscopic traffic model. On the other hand, the path-space Fisher Information Matrix, (pFIM) reduces model complexity and can even be used to control fidelity. This is achieved by eliminating unimportant model parameters or their combinations. This results in easier regression of parametric models with a smaller number of parameters.

The method reconstructs the Markov Chain and emulates the traffic dynamics through Monte Carlo simulations. We use the microscopic interaction model from [40] as a representative traffic flow model to illustrate this parameterization methodology. During the comparisons we use both synthetic and real, rush-hour, traffic data from highway US-101 in Los Angeles, California.
\end{abstract}

\section{Introduction}

Unlocking the hidden mechanisms behind a number of observed and still not well-understood traffic phenomena is one of the fundamental tasks in traffic science. In that respect modeling and simulation is paramount in resolving and understanding complex traffic dynamics. Testing and comparing results from simulations against reality is essential.

Traffic modeling and parameterization approaches have recently intensified due to the demand for faster and sometimes automatic parameter evaluation. The increasing deployment and testing of Vehicle Automation and Communication Systems (VACS) which is currently occurring promises to revolutionize the field and reshape the landscape of what is possible and what can be achieved [4]. With the advent of GPS enabled devises and a flood of incoming unordered traffic related data new methodologies must be developed which can quickly appraise and accordingly adjust the traffic model in order to best emulate the changing traffic conditions [10].

\footnotetext{
*Mathematical Sciences, Lund University, Lund, Box 118 SE-22100, Sweden

${ }^{\dagger}$ Department of Mathematics and Statistics, University of Massachusetts, Amherst, MA 01003
} 
Producing a successful such traffic model on the other hand involves, among other things, fitting the model to actual data and testing its performance under observed roadway conditions. Anyone who has tried to fit models to real data in order to emulate actual traffic conditions however knows well that this is a cumbersome task with non-unique solutions and can involve ad-hoc procedures. "Solving is often more of an art than a science", [8]. Not surprisingly methods which work well for obtaining parameters of linear systems may lead to substantial errors [22] when applied to linearized or nonlinear systems such as those typically comprising current traffic flow models.

Significant recent progress has been made in developing sensitivity analysis tools in areas such as chemical reactions, biological networks, mathematical finance, operations research etc. The reason for this wide spread appeal of sensitivity analysis is that it allows us to test model robustness as well as increase understanding of the relationship between input and output parameters. Sensitivity analysis furthermore simplifies regression. This is achieved by identifying and removing redundant model parameters or fixing parameters that have minor effect. A primary sensitivity test can ease the calibration stage by identifying and focusing on the sensitive parameters.

One of the many challenges within model parameterization is that the process depends strongly on the traffic situation as well as the data. If for instance the data involves congested traffic then parameters related to setting the free flow velocity would be unimportant. If instead we are looking at a junction with several entrances and exits lane changing parameters would suddenly become more important. This dependence on the data is discussed in detail in [44]. In general the traffic context is of great importance when parameterizing a traffic model and is one of the main reasons that dynamic parameterization is a very important and emerging area within traffic modeling.

Some of the most common approaches of parameter estimation include maximum likelihood, least squares, Bayesian cost method or Maximum-A-Posterior (MAP) to name a few. Typical mechanisms include log-likelihood methods and Girsanov transformations, $[11,28,32]$ or pathwise sensitivity methods, [35]. Many of the existing sensitivity analysis mechanisms however are usually associated with an overwhelming computational cost as the parameter space increases. Finite difference sensitivity analysis methods, [2, 33], for instance usually involve gradients and incur great computational costs as the number of parameters increases. The gradient-free information theoretic methods in [30] on the other hand can avoid these costs and in that respect the methodology we propose here stems from such an approach. Some related data fitting methods were also recently proposed in the context of coarse-graining of molecular simulations in [18].

Applying statistical mechanics in order to reconstruct the most likely parameter space for the traffic flow model is not an entirely new idea. Most of the proposed methodologies depend on knowing or assuming an equilibrium distribution of the system dynamics. [42] for instance use Markov chain Monte Carlo methods to sufficiently sample the parameter space and from that estimate the essential parameters for the traffic flow model. Their method is applicable to traffic flow models based on the discrete velocity equation. The approach focuses on reconstructing the values of the parameters comprising the flow-density relationship for that traffic flow model. In order to achieve this objective the authors built posterior distributions of the traffic flow model parameters related to the flow-density relationship. Subsequently an optimization problem needs to be solved in order to obtain the values of the parameters which reduce model sensitivity attributed to errors within the data. Success of this method therefore relies on assuming the correct form for the inverse crime [17] relationship as well as obtaining a solution to the optimization problem.

A slightly different, although common approach for statistical mechanics, is proposed in the traffic model by [26]. The authors put forth a method which estimates the state transition probability matrix of the Markov chain representing the dynamics of the traffic model. In order for this transition matrix to be constructed the authors must assume that the equilibrium distribution of the traffic dynamics is known in advance. The methodology 
can be applied even under limited information conditions such as partial traffic observations. This is therefore a very interesting approach due to the direct possible applicability under actual traffic conditions. The authors in [48] follow a similar approach for a stochastic macroscopic freeway network traffic model. Due to its macroscopic nature the model has the obvious advantage that a much larger network can be considered and quickly analyzed. This method can also be applied under sparse traffic data. Currently this method is based on a particular underlying stochastic model. Instead of making assumptions about the underlying equilibrium behind the traffic dynamics the authors assume knowledge of some of the important model parameters. This limitation however can be eased by implementing some of the methods proposed in [52].

A similar approach is followed by [34] where a new multi-lane macroscopic traffic model for highways is derived. Each lane is modeled individually with a first-order macroscopic traffic flow model and divided into segments. The model originates from the conservation equation and applies the cell transmission paradigm in order to properly transmit flow information between each segment within the same lane. This model is furthermore extended to account for lane changes by incorporating information about lateral and longitudinal interactions. Assumptions behind the model are reasonable and feasible throughout. Features such as the capacity drop or limited capacity off-ramps are incorporated. This model is then calibrated by minimizing a cost function which is comprised of vehicle speeds and longitudinal flow for specific roadway data from Australia. To produce the model parameters data from a number of different days are processed with an advanced optimal control method. The calibrated model is subsequently validated against actual traffic on that same roadway and shown to perform satisfactorily.

We propose an innovative approach here which can contribute an additional methodology to facilitate traffic flow model parametrization and the overall data fitting process. In particular we propose an adaptive methodology which obtains the values of the unknown traffic model parameters from partial traffic observations. We will show that this mechanism is fast making it possible for the model to regularly update the values of its parameters in order to make maximum use of available information and adapt to prevailing and changing traffic conditions. We will not need to train our method in advance in order to obtain the values of the unknown parameters. More specifically we develop two information theoretic tools, the relative entropy rate (RER) and the path-space Fisher information matrix (pFIM) which process traffic data as they become available in order to perform sensitivity analysis and obtain the model parameters.

In order to better understand some of the properties behind our parametrization approach we need to first clarify the concept of equilibrium dynamics from the perspective of statistical mechanics. In particular we will focus on the similarities and differences from the classical concept of Wardrop or user equilibrium (UE) that most researchers working within traffic modeling are accustomed to. One way to understand UE is by considering a traffic system where there does not exist any travelers who can improve their travel cost by changing their route. It is clear therefore that Wardrop equilibrium can also be understood in a game-theoretic sense (Nash-type).

In statistical mechanics however we talk about statistical equilibrium which is described by a probability distribution function (pdf) usually of unknown form-unless it is a Gibbs state and we have detailed balance. The precise definition of equilibrium in statistical mechanics is provided in equation (16) at Appendix B. In short, the system has reached equilibrium when equation (16) is satisfied which implies that a certain minimum "energy" state has been found. This is very much like the UE where a minimum cost has been found by the traffic system. But this is where the similarities end. In the statistical mechanics definition it is possible that, due to fluctuations and noise, the system may reach another state within the same equilibrium pdf as well. In fact there can be several different such equilibrium states for a given system which can result in vehicles adjusting their speeds and spacings from each other as they move from one such state to another. Thus choosing 
different routes is in fact possible under this concept, in contrast to UE. The probability distribution function already includes all the possible such routes with similar minimums and the stochastic dynamics allows the system to visit each of those. As a result the statistical mechanics definition of equilibrium is slightly more general than that of Wardrop equilibrium. We refer also to Appendix B for further discussion and relevant definitions.

The significant difference of our approach is that we propose tools which can extract statistical properties even from non-equilibrium dynamics in order to determine the best possible model under the current traffic conditions. As a result the traffic model is dynamically adjusting to prevailing conditions. The obvious advantage therefore over existing methodologies is that we do not need to assume the existence of an equilibrium distribution $\pi$ (16) for the traffic dynamics. The main challenge, which usually hampers most modeling approaches and parameterization methodologies is to detect whether the system is in equilibrium and then to find out what that equilibrium is. We propose a parameterization methodology here which avoids both of these issues. We therefore do not require that the system is in equilibrium or assume that such an equilibrium may exist at all. It is therefore possible to apply our method and establish the appropriate traffic model parameters even if the system dynamics are changing.

When dealing with the classic problem of parameter estimation success relies on understanding concepts such as bias and efficiency of the resulting estimator. We identify these concepts and highlight their relation in terms of information theory here. Parameter estimation is successful if the value of the estimated parameter $\hat{p}$ approaches the value of the true parameter $p^{*}$ with a minimum error. More specifically we say that the estimator is asymptotically unbiased if $\mathbf{E}[\hat{p}] \rightarrow p^{*}$ as the number of data points approaches infinity. Furthermore we say that the estimator is efficient if its covariance matrix $S_{\hat{p}}$ is a minimum. It is important to note that efficiency is measured relative to the maximum information available in the data. The methodology proposed here is able to identify the model parameters in such a way which guarantees that as much as possible information has been extracted from the available traffic data. Efficiency lower than $75 \%$ for instance is known to be responsible for serious errors in the estimated parameters, [15].

We apply the following strategy in order to leverage current traffic information and establish an unbiased and efficient [18] model under evolving conditions:

- Reduce the model by eliminating unimportant parameters (or their combinations) as indicated by pFIM.

- Subsequently compute the path-space (time series) RER in order to explore the parameter space and identify the most sensitive parameters.

- Update these parameters in order of importance as indicated by RER. In that respect there are two alternatives: a) update value of parameter as indicated by the perturbation and its result on RER or b) perform complete optimization. We refer to Section 5 for details on these two options.

The methodology is general and can therefore be applied for any underlying microscopic traffic flow model such as for instance the well-known Cellular Automaton (CA) type model in [27] or the car-following type model in [43]. All that is required from the microscopic model is that a formulation of rate or probability for vehicle motions will be provided. Specifically, in the case of the CA model by Nagel-Schreckenberg [27] the needed rate is given by equation (5) within that manuscript,

$$
W[-\sigma(i),-\sigma(i+1) \mid \sigma(i), \sigma(i+1)]=\left(1-p^{*}\right) \frac{1+\sigma(i)}{2},
$$

which describes the transition probability to move a vehicle from location $i$ to location $i+1$. The spin-like variable $\sigma(i)$ appearing in the equation above can take values \pm 1 indicating 
a vehicle or not at a given location $i$ (see (1)) while the probability $p^{*}$ is just a previously chosen randomization parameter. In the case of car-following models the rate function is given by the corresponding acceleration function related to the underlying optimal velocity model. Specifically the rate function in the case of the car-following model by [43] is the rather elaborate acceleration function $\dot{v}_{\alpha}$ described in equation (6) therein. Similar such functions can be provided for the well-known car-following models by Newel or Bando et al in equations (4) or (5) respectively both of which can be found in [43].

In this article we use the stochastic model by [40] as the underlying traffic flow model. This also allows us to provide a complete description of the processes as well as concrete numerical comparisons against data. The model by [40] is a microscopic multi-lane traffic flow model which incorporates multi-class interactions with both lateral and longitudinal considerations through a local asymmetric look-ahead potential. This model has been extensively tested and validated in more than 46 mathematical and traffic related publications by a number of authors. The dynamics are built upon basic statistical mechanics principles by putting together an Arrhenius law for the motion of vehicles as well as an advanced "look-ahead" behavior [37] incorporated through an Asymmetric Simple Exclusion Process (ASEP) [41]. This model is subsequently simulated through a kinetic Monte Carlo [5] or Gillespie algorithm.

We begin in Section 2 by providing the general framework of our approach and notation to be used. In that same section we also provide the details of the microscopic traffic flow model from [40] which we will use for our simulations and subsequent comparisons. Then in Section 3 we introduce the first information theoretic tool, the relative entropy rate, which will assist in appraising model parameter sensitivities. In Section 4 we produce the second information theoretic tool, the Fisher information matrix, which will be helpful in reducing traffic model complexity by eliminating the unimportant model parameters. We follow up with numerical results under synthetic data of how such tools can be applied to produce the needed values for the traffic flow model parameters in Section 5. Both of the information theoretic tools RER and pFIM are then illustrated against real traffic data in Section 6 . We end with a short discussion in Section 7. The Appendix is used to outline some of the background related to information theory and its use within statistical mechanics in order to ascertain differences between stochastic measures via the relative entropy semi-metric.

\section{A microscopic traffic flow model}

We now present the underlying traffic model from [40] and [1] which will be used to showcase our parametrization approach. In the subsequent Sections 3 and 4 we will built the necessary, information theoretic, infrastructure around this underlying traffic flow model and will construct the relevant parametrization tools. As already discussed in the introduction any Cellular Automaton (CA) traffic flow model such as those in $[27,45,13]$ or other type stochastic model $[24,19,38]$ can be parametrized under the proposed methodology which we will present in Sections 3 and 4.

We define the roadway as a two-dimensional lattice $\mathcal{L}$, which is partitioned into $n \times m$ cells. In a multi-lane environment each cell location $i \in \mathcal{L}$ will consist of two indexes, $i=\left(i_{x}, i_{y}\right)$ which denote the position along the highway, via $i_{x}$ and the position based on lane number, via $i_{y}$. Each cell is assumed to be approximately 6 meters in length and can accommodate a vehicle plus minimum gap. On each lattice cell $i \in \mathcal{L}$ we define an order parameter,

$$
\sigma(i)= \begin{cases}1, & \text { if a vehicle occupies site } i \\ 0, & \text { if site at } i \text { is empty (no vehicle). }\end{cases}
$$

A configuration $\sigma=\{\sigma(i): i \in \mathcal{L}\}$ therefore is an element of the configuration space $\Sigma=\{0,1\}^{\mathcal{L}}$. We use $\left\{\sigma_{t}\right\}_{t \geq 0}$ to denote the stochastic process on $\mathcal{L}$ whose dynamics, from [1] or [40], we provide below. 
Note that the system can be in any of $2^{|\mathcal{L}|}$ possible states. Vehicle interactions and evolution between each of those states is dictated by the following spin-exchange, [23] Arrhenius dynamics,

$$
r(i, j, \sigma)=c_{0} \sigma(i)(1-\sigma(j)) \exp (-U(i, j, \sigma)), \quad \text { for } j_{x} \geq i_{x},
$$

where $i$ and $j$ are nearest neighbor locations in $\mathcal{L}$. Here $i_{x}$ and $j_{x}$ denote the longitudinal index, along the roadway. Under such dynamics a spin-exchange between nearest neighbor cells $i$ and $j$ implies that the system will allow the order parameter $\sigma(i)$ at cell location $i$ to exchange value with the one at location $j$. Note that the exchange can only take place if the location at $i$ is occupied and the one at $j$ is not. This is interpreted as moving a vehicle from location $i$ to $j$. At the same time it is also impossible for collisions to occur since if both locations are occupied with $\sigma(i)=1$ and $\sigma(j)=1$ the corresponding rate in (2) will be 0 .

The parameter $c_{0}$ in (2) represents the characteristic or relaxation time for the stochastic process and is directly linked to how quickly vehicles perform moves from cell to cell. This parameter will be calibrated based on our proposed methodology in Sections 3 and 4 . For one-lane roadway $j=i+1$ since the exchange occurs only for nearest neighbors and $j$ ahead of $i$. The interaction potential $U$ in (2) regulates how strongly vehicles interact with each other and will be fully presented below in Subsection 2.1 while the multi-lane environment will be provided in Subsection 2.2.

\subsection{Interaction potential}

The interaction potential $U(i, j, \sigma)$ in (2) is comprised of contributions from short range interactions $U_{e}$ as well as anisotropy interactions $U_{\alpha}$ which we will provide in Subsection 2.2. As a result $U(i, j, \sigma)=U_{e}(i, \sigma)+U_{\alpha}(i, j, \sigma)$, [23]. The short range interactions $U_{e}$ are computed from,

$$
U_{e}(i, \sigma)=\sum_{\substack{j \in \mathcal{L}, j \neq i \\\left|j_{x}-i_{x}\right| \leq L}} J(i, j) \sigma(j)
$$

where $J$ is the local interaction potential function and will be provided below in (4). Note that the effect of the interaction potential $U_{e}$ is to appraise the number of vehicles up to a distance $L$ around the current vehicle. We usually take $L=3$ cells. Since the overall impact of this potential on the rate (2) is as a penalty term then the rate (or probability) to move towards locations with lower number of vehicles will be favored.

Specifically the local interaction potential $J$ in (3) is defined as follows,

$$
J(i, j)=\frac{1}{L} W\left(\frac{j_{x}-i_{x}}{L}\right), \text { and } W(z)= \begin{cases}J_{0}, & \text { for } 0 \leq z \leq 1 \\ 0, & \text { otherwise }\end{cases}
$$

Note that this definition introduces asymmetric one-sided interactions since $W(z)$ is nonzero only for positive and therefore forward range, $0 \leq z \leq 1$. Furthermore, based on this definition, we note that the interactions are local since each vehicle interacts with only a limited number of vehicles up to a distance $L$ ahead. This is therefore how the vehicle lookahead property is realized. The parameter $J_{0}$ relates to how strongly vehicles interact with each other and will be calibrated based on our proposed methodology in Sections 3 and 4.

The stochastic process $\left\{\sigma_{t}\right\}_{t \geq 0}$ constructed above is a continuous time, jump, Markov process on $L^{\infty}(\Sigma ; R),[1,23]$. In statistical mechanics a process similar to the one constructed above is known as an asymmetric simple exclusion process or ASEP, [41]. The probability of a spin-exchange from location $i$ to the location $j$ during time $[t, t+\Delta t]$ is, $[40,23]$,

$$
r(i, j, \sigma) \Delta t+O\left(\Delta t^{2}\right) .
$$




\subsection{Multi-lane environment}

In Section 2.1 we established the main machinery which allows vehicles to move on a roadway based on local traffic conditions. We now establish a specialized mechanism from [1] which will allow vehicles to better appraise and efficiently utilize empty space in a specific lane of a multi-lane road. In order to better control this lane-changing process we first define the following parameters, $c_{r}, c_{l}$ and $c_{f}$ for moving the vehicle to the right lane, the left lane or forward respectively.

Specifically the lane changing mechanism is incorporated via the anisotropy potential,

$$
U_{\alpha}(i, j, \sigma)=\sum_{\substack{j \in \mathcal{L}, j \neq i \\ 0 \leq j_{x}-i_{x} \leq L}} \phi(i, j) \sigma(j) \text { where } \phi(i, j)= \begin{cases}c_{l}, & \text { if } j_{y}=i_{y}+1 \text { (left lane), } \\ c_{r}, & \text { if } j_{y}=i_{y}-1 \text { (right lane) } \\ c_{f}, & \text { if } j_{y}=i_{y} \text { (forward). }\end{cases}
$$

This anisotropy potential also acts as a penalty term penalizing each lane depending on how many other vehicles occupy that lane. The more vehicles occupy a given lane then the higher the penalty would be which in turn translates to a proportionally reduced rate $r(i, j, \sigma)$ and overall chance (5) for the vehicle to move to that lane.

The above multi-lane construction makes it possible to easily simulate a number of different lane changing strategies. We can for instance allow drivers to mainly use the left lane for passing by making the constant $c_{l}$ slightly larger (bigger penalty) than the other two constants. Thus vehicles would mainly prefer to use their current lane or the right lane instead of moving to the left lane, assuming that all other conditions are equivalent. Alternatively if we allow $c_{l}=c_{r}$ we would enforce equal rates for passing which is equivalent to the USA paradigm allowing vehicles to pass on either lane. Clearly all these parameters will be calibrated by our novel methodology in Sections 3 and 4 . We follow ideas from [29] and [36] in terms of developing schemes which agree with observations of lane changing behavior.

In our simulations, three different motions were permitted: left, right and forward. Nevertheless the definitions can be very easily adapted to allow other exchanges, such as diagonal moves by addition of two more parameters, although the value of this extra possibility may not be substantial. This can be understood as a vehicle advancing forward and switching lanes simultaneously.

\subsection{Spin-flip Arrhenius dynamics}

In this model we can implement both spin-flip and spin-exchange Arrhenius dynamics. The spin-exchange mechanism introduced above in (2) is responsible for motion of vehicles within the roadway. The spin-flip mechanism on the other hand models how vehicles interact while entering or exiting the roadway. During a spin-flip the system will actually allow the order parameter $\sigma(i)$ at location $i$ to change sign. Note however that this move does not reciprocate by exchanging spins between two locations as the spin-exchange mechanism does. This new spin-flip mechanism is interpreted instead as placing (removing) a vehicle at (from) a specific location $i$ if $\sigma(i)=0(1)$. This mechanism is only active at specific locations $i^{*}$ on the road where entrances and exits are marked.

The rate at which a process will exit or enter the roadway is given by the following spin-flip Arrhenius dynamics, [1],

$$
r^{s f}(i, \sigma)= \begin{cases}c_{a} e^{-U_{s f}(i, \sigma)}, & \text { when } \sigma(i)=0, \\ c_{d}, & \text { when } \sigma(i)=1,\end{cases}
$$

where $c_{a}$ and $c_{d}$ are related to the frequency vehicles are added or removed from the roadway 
and must be calibrated from data. The potential function in (7) is given from,

$$
U_{s f}(i, \sigma)=\sum_{\substack{j \in \mathcal{L}, j \neq i \\|j-i| \leq L}} J(i, j) \sigma(j) .
$$

The probability of spin-flip at $i$ during time $[t, t+\Delta t]$ is, $r^{s f}(i, \sigma) \Delta t+O\left(\Delta t^{2}\right)$. In terms of traffic this is understood as the probability that a vehicle will enter/exit the highway at $i$.

Other situations including external events can be modeled via the addition of an external potential $h$ in the potential $U$ at (2) which is location and even time specific $h \equiv h(k, t)$. In this case we can simulate events related to bad weather or accidents influencing traffic on the roadway at specific locations and for a limited duration.

The multi-class implementation which is able to accommodate vehicles of different types is possible by applying different conditions on model parameters depending on vehicle type [1]. Typically, for instance, we provide a longer look ahead rule $L$ for trucks, since they can actually detect traffic much further ahead of them than other vehicles can. Similarly we have the possibility to allow them to occupy from 2 to 4 lattice cells during the simulation. We can also calibrate the characteristic time parameter $c_{0}$ and strength of interactions $J_{0}$ in order to better match the respective speeds for trucks and their slower way of interacting with other smaller vehicles around them. Typically, for trucks for instance, the $c_{0}$ will be lower than that for passenger vehicles since in general trucks drive at a slightly lower speed than other vehicles. This will result in a lower chance for a truck to move to an empty cell ahead when compared with a passenger vehicle (in the free-flow case).
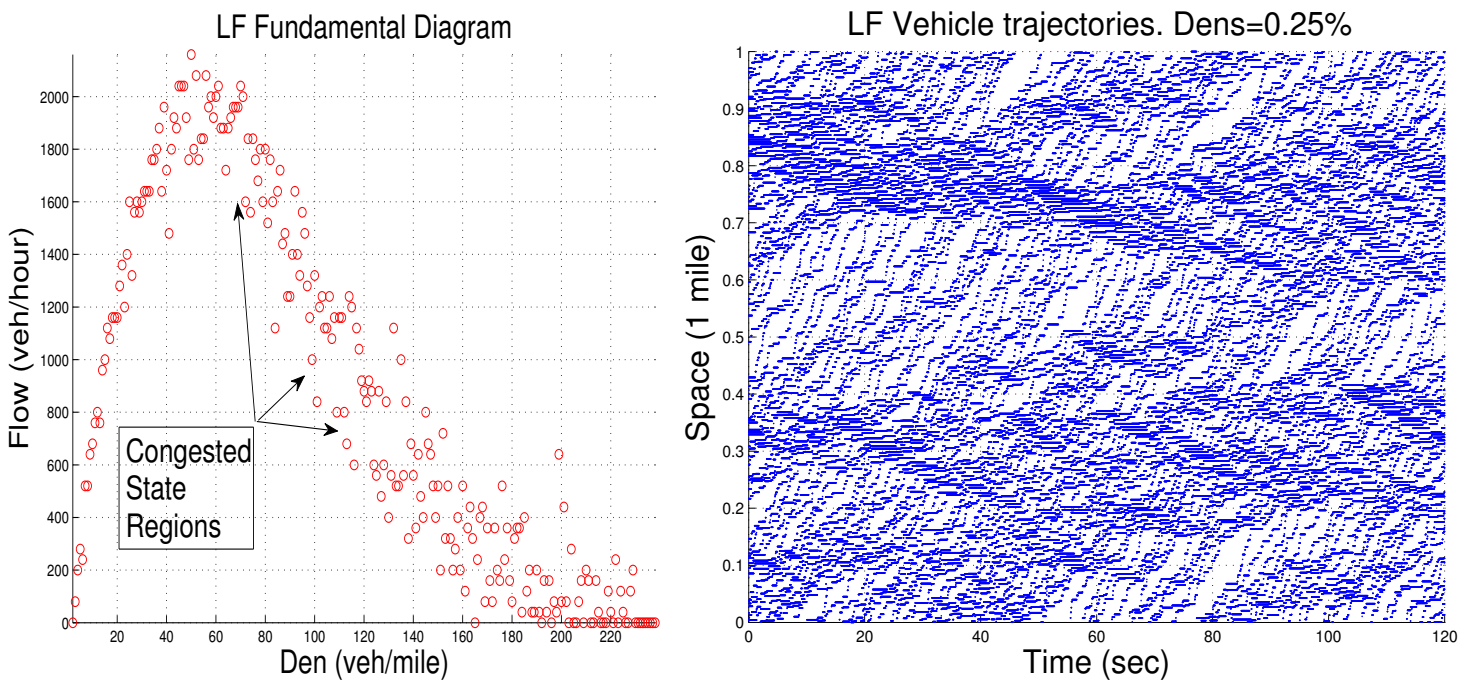

Figure 1: Traffic model simulation from synthetic data for a 2-lane, 1-mile long circular roadway with no entrances or exits. Model parameters are calibrated as in [7]. Cell size is approximately 6 meters. Left: the fundamental diagram. Note qualitative features such as the congested state regions as concentration increases above critical density. Correct quantitative features are also evident since the maximum flow is listed to be no more than 2200 vehicles per hour which is in agreement with experimental observations for a 2-lane roadway. Right: a 2 minute time/space section of vehicle trajectories near the critical density (vehicle concentrations $=25 \%$ ). We observe features such as shocks, rarefactions as well as retarded acceleration and timely braking.

Some of the most important model parameters such as $c_{0}$ and $J_{0}$ are calibrated so that the following rules are followed: a) the average speed of a vehicle with no other vehicles around 
should be approximately 65 miles/hour and b) the speed of propagation for any backward moving wave (driver reaction times) should be approximately 10 miles/hour $[49,45]$. See $[40,7]$ for information on how exactly these criteria are used in order to calibrate these as well as the remaining model parameters. The resulting simulation under these model parameters is provided in Figure 1 above. A number of important features which agree with experimental observation are evident in this figure. We observe congested state regions above critical density as well as the correct values for the maximum flow for this 2-lane roadway. Furthermore vehicles do not stop or start instantaneously as can be seen from the time/space trajectories in that figure. This has been shown [40] to be attributed to the look-ahead feature in the interaction potential (4) which is responsible for slowly speeding up vehicles when sufficient empty space is discovered ahead or alternatively slowing them down when reaching a queue up front. Overall therefore the current microscopic model seems to reproduce a number of key observational features we are accustomed to from actual traffic.

For more details on rates, such as (2) and potential functions such as (3) or the overall stochastic dynamics and Monte Carlo simulation we refer to [7, 40]. Other important attributes of this model include:

- Model is consistent with the Lighthill-Whitham-Richards in the case a single vehicle class as shown in [40].

- Anisotropic interactions which can differ for each vehicle class by adjusting $J_{0}$ in (4).

- Model is well posed [10] as long as there exist at least a single vehicle on roadway.

- A version of the same traffic flow model is applicable for the case of lattice-free domains and therefore vehicles of different sizes occupy different spaces [38, 39].

- This later version of the traffic flow model allows small vehicles to move even when larger ones are stopped [10].

\section{Information theoretic modeling approach}

In this section we built the mathematical tools which quantify information loss in the space of time series (path space). As indicated in the introduction information content within the data is linked to estimator efficiency. Lack of estimator efficiency is responsible for serious errors in the estimated parameters and resulting model.

Let us first define the vector $p=\left(p_{1}, p_{2}, \ldots, p_{K}\right)^{T}$ which contains all of the unknown traffic model parameters to be determined (here $v^{T}$ denotes the transpose of vector $v$ ). In the traffic flow model presented in Section 2 for instance we let $p=\left(c_{0}, J_{0}, c_{r}, c_{l}, c_{f}\right)^{T}$. We also simplify the notation for the rates in $(2)$, to just $r_{k}(\sigma) \equiv r(i, j, \sigma)$ where $k$ indexes through all the rates from locations $i \in \mathcal{L}$ to their three nearest neighbors. Furthermore to emphasize the dependence of the rate on the parameter vector $p$ we now explicitly write $r_{k}(\sigma ; p)$ instead of $r_{k}(i, j, \sigma)$.

We will construct an approach which will improve the values for those parameters based on appraising the current traffic situation and reducing the information loss. We note that many of those parameters can only take a certain range of values in order to remain physically relevant for modeling vehicles within a roadway and not rockets in space for example. In that respect therefore the final value chosen to be used for a given parameter may not be the optimal one if that optimal is not physically relevant. In that case the value chosen is the one nearest the optimal and still within the allowed range for that parameter. The range within which the parameters will remain physically relevant is known in advance during the calibration of the model as for instance in [40].

Let us now define a perturbation vector $\epsilon_{*}$ which will successively perturb each and every one of the parameters in the parameter vector $p$ in order to optimize the model. For instance 

.01 .

Following the general information-theoretic perspective from [8] and [20] and specifically the introduction of the relative entropy rate (RER) as a sensitivity analysis tool in [31] we can define below one of the estimators for RER which will facilitate appraising traffic information. We provide the theoretical background behind this estimator in the Appendix. Following (12) we define the estimator for the RER over time $T$ for a general traffic flow model given a perturbation $\epsilon_{*}$ on the parameter vector $p$ as follows [30],

$$
R_{r}\left(\epsilon_{*}\right)=\frac{1}{T} \sum_{i=0}^{N} \Delta t_{i}\left[\sum_{k=1}^{M} r_{k}\left(\sigma_{i} ; p\right) \log \frac{r_{k}\left(\sigma_{i} ; p\right)}{r_{k}\left(\sigma_{i} ; p+\epsilon_{*}\right)}+r\left(\sigma_{i} ; p+\epsilon_{*}\right)-r\left(\sigma_{i} ; p\right)\right] .
$$

We note ${ }^{1}$. Here we use the index $k$ to count through all the possible rates (to move left, right or forward) for all cells in the lattice. We let $r\left(\sigma_{i} ; p\right)=\sum_{k=1}^{M} r_{k}\left(\sigma_{i} ; p\right)$ denote the sum over all rates. We note that the total number of rates $M$ will vary as vehicles move within or exit/enter the roadway. That number $M$ however will always be finite since the maximum number of vehicles that can occupy the roadway is $\mathrm{nm}$ and the maximum $M$ is $3 \mathrm{~nm}$. As a result the total quantity $r$ will always be well-defined.

To present how the parameterization method works we use the traffic flow model [40] as outlined in Section 2. The synthetic data used for this example emulates a section of a highway without entrances or exits. We compute how the RER changes over time after perturbation of a given model parameter. Specifically we present five such different parameter perturbations and display their pathwise RER timeseries in Figure 2. Vehicles enter randomly on one side of the highway and subsequently are removed when reaching the end of that highway. Each of the five timeseries provided in Figure 2 corresponds to a perturbation by $+10 \%$ while keeping the other four parameters fixed to their original values. The parameters used are: $c_{0}, c_{r}, c_{l}, c_{f}$ and $J_{0}$. Specifically $J_{0}$ is related to how strongly vehicles interact with each other while $c_{l}, c_{r}$ and $c_{f}$ relate to the strength of vehicle to vehicle interactions while changing to the left lane, changing to the right lane or moving forward respectively. Finally $c_{0}$ represents how quickly moves are performed and therefore is directly coupled to vehicle velocities. Perturbations are in the order of $\pm 10 \%$ so that we will be able to compare the resulting RER even if the parameters themselves differ substantially in value. In Figure 2 only the perturbations by $+10 \%$ are presented. See [3] for a study on the effects related to the size of the perturbation.

Figure 2 represents the sensitivity analysis for each of those parameters according to RER. According to these results the parameter for moving forward, $c_{f}$, is the most important or most sensitive for this traffic model based on the given data. Thus $c_{f}$ is the first parameter we should attempt to change the value of in order to improve the performance of our model. The amount these parameters must be adjusted by is already indicated by the perturbation and its result on the RER. Specifically if the particular perturbation produces an improvement on the RER then we simply accept that new value for that parameter without any additional computational cost. Otherwise we do not alter the value of the parameter. Alternatively we can perform a complete optimization in order to discover the best values for those parameters as will be illustrated in Section 5. The difference between the two approaches involves questions about available computational resources as well as possible time limitations (real-time computations versus off-line processing). We provide further comments about such questions in Section 5 as well as in the conclusions.

We point out that the relative entropy and maximum likelihood are intimately related as is well-known from the statistics literature [50] (Section 9.5). By analogy (9) employs instead of relative entropy, the relative entropy rate. Note that if the transition probabilities

\footnotetext{
${ }^{1}$ To make the above formula easier to read on paper we write $\sigma_{i}$ instead of $\sigma_{t_{i}}$. To clarify the notation therefore and avoid confusion we point out that $\sigma_{i}$ denotes the configuration at time $t_{i}$ while $\sigma(i)$ keeps track of whether a vehicle exist at location $i$ or not.
} 
RER for 5 parameters

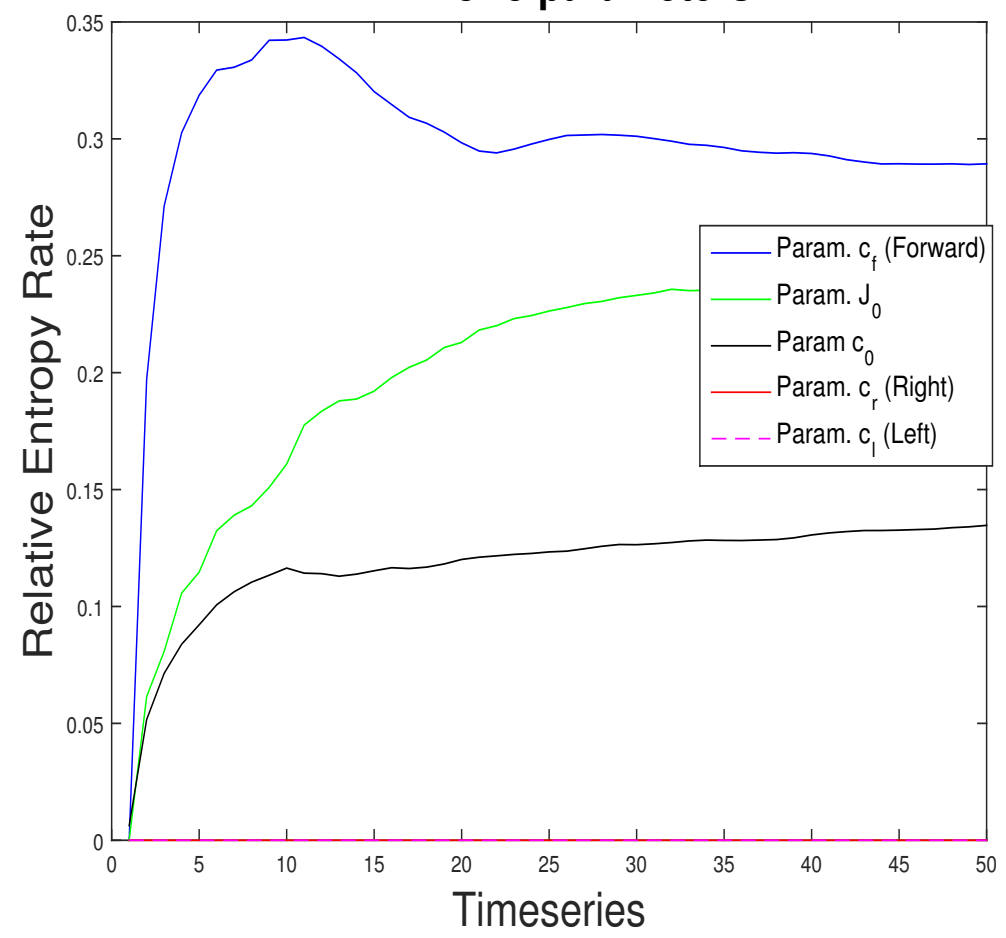

Figure 2: The RER, $R_{r}$ versus time of each of 5 model parameters is presented. We perturb one parameter at a time by $+10 \%$ while keeping all the others fixed. Synthetic data is used for the simulations. Sensitivity analysis indicates that the parameters whose values must be adjusted in that model, in order of importance, are: $c_{f}$ followed by $J_{0}$ and $c_{0}$. The parameters $c_{r}$ and $c_{l}$ are almost 0 and therefore do not need to be further adjusted. This simple computation therefore indicates which parameter values are most important for the model and in which direction they must be adjusted $( \pm 10 \%)$. We used synthetic data and the model in Section 2 as the underlying traffic model.

in (9) are replaced with a stationary distribution and corresponding independent samples then (9) becomes equivalent to the classical maximum likelihood principle. In this sense (9) is equivalent to a maximum likelihood principle for a correlated time series. We refer to Section III.B of [18] for a complete discussion.

Finally, (9) provides a pathspace variational inference principle that is a direct analogue with recent equilibrium variational inference methods in machine learning [25]. See [12] for time series data and non-equilibrium problems.

\section{Fisher information matrix}

To further reduce model complexity and computations while obtaining the traffic flow model parameters we now introduce an additional tool.

Following information theoretic methodology as in [30], or (15) in the Appendix, we can now define an estimator for the RER associated pathwise Fisher information matrix (pFIM),

$$
F_{n}=\frac{1}{T} \sum_{i=0}^{N} \Delta t_{i} \sum_{k=1}^{M} r_{k}\left(\sigma_{i} ; p\right) \nabla_{p} \log r_{k}\left(\sigma_{i} ; p\right) \nabla_{p} \log r_{k}\left(\sigma_{i} ; p\right)^{\mathbf{T}}
$$


where the $\mathbf{T}$ above denotes the transpose. Equation (10) allows us to further quantify individual parameter sensitivity. The significant difference of this pFIM against other equivalent methods is that it constitutes a gradient-free approach [30] since it is constructed from explicit formulas which depend only on the propensity functions (2) with respect to the parameter vector $p$. As a result no complicated gradients need be computed at each time step and the overall computational overhead is considerably reduced.

Table 1: The traffic flow model rates and their derivatives for $p=\left(c_{0}, J_{0}, c_{r}, c_{l}, c_{f}\right)^{T}$.

\begin{tabular}{|c|c|c|}
\hline & Rate $r_{k}\left(\sigma_{i} ; p\right)$ & $\nabla_{p} \log r_{k}\left(\sigma_{i} ; p\right)$ \\
\hline Forward motion & $c_{0} \sigma_{i}\left(1-\sigma_{i}\right) \exp \left[-U_{e}\left(J_{0}\right)-U_{\alpha}\left(c_{f}\right)\right]$ & $\left(\frac{1}{c_{0}},-\frac{U_{e}}{J_{0}}, \quad 0, \quad 0,-\frac{U_{\alpha}}{c_{f}}\right)^{T}$ \\
\hline Left lane motion & $c_{0} \sigma_{i}\left(1-\sigma_{i}\right) \exp \left[-U_{e}\left(J_{0}\right)-U_{\alpha}\left(c_{l}\right)\right]$ & $\left.0,-\frac{U_{\alpha}}{c_{l}}, \quad 0\right)^{T}$ \\
\hline Right lane motion & $c_{0} \sigma_{i}\left(1-\sigma_{i}\right) \exp \left[-U_{e}\left(J_{0}\right)-U_{\alpha}\left(c_{r}\right)\right]$ & $\left(\frac{1}{c_{0}},-\frac{U_{e}}{J_{0}},-\frac{U_{\alpha}}{c_{r}}, \quad 0\right.$ \\
\hline
\end{tabular}

We provide an example of the computation involved in the pFIM based on the traffic flow model from Section 2. Specifically we compute the pFIM for the following parameter vector $p=\left(c_{0}, J_{0}, c_{r}, c_{l}, c_{f}\right)^{T}$ on the rates allowing vehicles to move left, forward or right. We display these rates $r_{k}$ and corresponding derivatives $\nabla_{p} \log r_{k}$ in Table 1.

Furthermore performing spectral analysis on the pFIM we can easily discover the sensitive directions in the parameter space for our model. The most sensitive directions correspond to higher eigenvalues and visa-versa. The structure of the pFIM decouples interdependencies between parameters and provides all essential information about parameter sensitives within its diagonal entries (see Figure 3). This is a benefit over other similar pFIM methods such as for instance the linear noise approximation (LNA) based pFIM (see Figure 4 in [31]). In the LNA approach we obtain significant contributions in most entries of the pFIM. However the LNA is only an approximation of the microscopic model that breaks down at low densities. Hence it can (and will) yield wrong sensitivity results. This produces inconclusive sensitivity analysis and results in models which contain many more parameters, [21], than the method we propose.

Note that since the pFIM does not depend on the perturbation $\epsilon_{*}$ it has a computational advantage over the RER. Based on the pFIM we can immediately reduce our traffic flow model by removing some of the less important parameters before performing any parameter perturbations. According to the pFIM a parameter is judged to be less important in terms of the amount of information it conveys to the model. This is easily quantified in the pFIM through those entries in that matrix which are zero or near zero. This is for instance the case for the parameters $c_{f}, c_{r}$ and $c_{l}$ under the synthetic data presented in Figure 3 (different data from those in Figure 2). In Figure 3 the parameters for which the pFIM is equal to zero can be eliminated. Model parameters which are important are associated with larger diagonal elements in the pFIM. Thus one significant benefit of the method proposed here is that it uncouples the parameter correlations since the off-diagonal elements are zero making it easier to evaluate their importance and true influence on the model.

An interesting question perhaps would be whether the information contained within pFIM is sufficiently important. Clearly using pFIM for models with several parameters is a definite advantage. However there are more reasons for using pFIM:

- Considering complex models with many more mechanisms (many lanes, entrances/exits, traffic lights, etc) since such a model would shrink or expand accordingly as traffic conditions change.

- Control fidelity of parametrization and understand variability and robustness of our model for the given conditions. The pFIM can produce error bars for the data assimilation/fitting step as can be seen in Section 5 . 
Figure 3: Path-wise pFIM sensitivity. In this example from synthetic data (different data than that presented in Figure 2) parameter $J_{0}$ is found to be most sensitive followed by $c_{0}$. Note that parameters $c_{f}, c_{r}$ and $c_{l}$ are near zero and therefore not as important in this traffic flow model. This profile however can change and the parameter space must be probed periodically, depending on the evolving dynamics provided within the traffic data. Furthermore note from (2) and (6) that the contributions from $c_{0}$ and $c_{f}$ are in some cases similar and almost redundant. This may explain why, according to pFIM and this synthetic data, $c_{f}$ is unnecessary and can be eliminated.

Finally we note that since pFIM is gradient-free the proposed sensitivity analysis is significantly faster when dealing with complex systems and/or large number of parameters as is usually the case for traffic models in a network environment. Furthermore the knowledge of the structure of the pFIM allows us to better identify important parameters, produce estimates for those parameters and test their robustness. Equivalently it is observed that parameters which correspond to faster reactions are more sensitive in a pathwise entropy as also observed in [31].

\section{Parameterizations of controlled fidelity through in- verse dynamic Monte Carlo}

The RER methodology introduced in the Section 3 can be exploited in order to update and improve the values of the model parameters in real time as the dynamics evolve on the roadway. If desired however RER can also be used to obtain the optimal such values for the model parameters. We provide an algorithm here which can accomplish this task. We note however that in many cases the actual optimum may not always be relevant or even necessary to be found. We discuss and clarify these points below.

In this section we outline an optimization procedure which allows to obtain the best values for our most important traffic flow model parameters based on properties and information contained within the RER. A similar study was curried out in a general mathematical context in [18] in the case of coarse graining microscopic dynamics.

There are a number of different approaches which allow us to achieve this result. We will use entropy based criteria in order to obtain the best approximation. Based on (13) we consider the minimization of RER as in [18]. Theoretically we can obtain the best value of 
a given parameter vector $p$ by minimizing RER, (9), as follows,

$$
p^{*}=\arg \min _{p}\left\{R_{r}\left(\epsilon_{*} ; p\right)\right\},
$$

where vector $p$ contains the previously identified from RER sensitive model parameters. The vector $p$ may include all such parameters or just a subset of the most important model parameters (i.e above some given RER threshold value).

The values allowed for the parameter vector $p$ are restricted within a range in order for the parameters to remain physically relevant. The optimality condition here is $\nabla_{p} R_{r}\left(\epsilon_{*} ; p\right)=0$ and is always achievable due to the convexity in $R_{r}$ with respect to the parameter vector $p$, as has been shown in [18]. See also Figure 4 here where the convexity is displayed numerically under synthetic data. The corresponding minimization scheme therefore is provided by,

$$
p^{n+1}=p^{n}-\frac{\alpha}{n} G^{n+1},
$$

where $n$ is just an index, $\alpha>0$ and $G^{n+1}$ is a suitable approximation of the path-space gradient $\nabla_{p} R_{r}\left(\epsilon_{*} ; p\right)$. Specifically $\nabla_{p} R_{r}\left(\epsilon_{*} ; p\right)=E\left(\left[G^{n+1} \mid G^{0}, p^{0}, \ldots, G^{n}, p^{n}\right)\right.$ where for this algorithm to work well we must obtain reliable estimates of the sequence of the gradient estimates, $G^{n}$. One way to achieve this and also increase the convergence of that sequence is to apply the Newton-Raphson method. In this case we choose the vector $G$ via,

$$
G^{n}=\operatorname{Hess}\left(R_{r}\left(\epsilon_{*} ; p^{n}\right)\right)^{-1} \nabla_{p} R_{r}\left(\epsilon_{*} ; p^{n}\right),
$$

where the Hessian is the FIM. Clearly using such an advanced method which involves the computation of the Hessian will result in an additional computational cost but at the same time it also provides quicker convergence (otherwise simpler alternatives without the Hessian could be considered instead). Following this procedure we can compute both the Hessian as well as the first derivative of $R_{r}$ by employing the ergodic properties of the RER. Specifically,

$$
\begin{aligned}
\operatorname{Hess}\left(R_{r}\left(\epsilon_{*} ; p\right)\right) & =-E_{\mu}\left[\sum_{k=1}^{M} r_{k}(\sigma ; p) \nabla_{p}^{2} \log r_{k}\left(\sigma ; p+\epsilon_{*}\right)\right] \\
\nabla_{p} R_{r}\left(\epsilon_{*} ; p\right) & =-E_{\mu}\left[\sum_{k=1}^{M} r_{k}(\sigma ; p) \nabla_{p} \log r_{k}\left(\sigma ; p+\epsilon_{*}\right)\right] .
\end{aligned}
$$

We numerically present the convexity property of RER as well as the existence of an optimal value in Figure 4. According to the sensitivity analysis curried out on the previous section and presented in Figure 2 the value of the parameter $c_{f}$ must be adjusted first. In Figure 4 therefore we explore the parameter space for $c_{f}$. The perturbations used are in the order of $+10 \%$ of $c_{f}$. The convexity property of RER is evident in that figure. Some of the noise displayed there is attributed to the noise of the stochastic dynamics. Based on the RER for the data the optimum value for $c_{f}$ is .5. It is also seen from that figure that most values in the range $.1 \leq c_{f} \leq 2$ would also produce satisfactory results while keeping the loss of information low.

Parameter values however must have physical relevance since they could directly or indirectly influence vehicle speeds or driver reaction times etc. It is therefore not always possible that the actual optimum can be used. In that case the value of the parameter nearest the optimal and still within the range of physical relevance should be used instead. We overlay, as an example, in Figure 4 the range of physical relevance for $c_{f}$ in red as well as the best value, marked in green, for this parameter within that range. Thus $c_{f}=.6$ is chosen based on the analysis from the RER of the given traffic data.

Note that the gain due to information quantification is evident here. The value of $c_{f}$ based on empirical calibration without the use of the information theoretic approach 
Figure 4: RER, information theory based parameter perturbation and optimization. In this example parameter $c_{f}$ is perturbed by $10 \%$ and its optimum is found near .5 . The range of physical relevance for this parameter is represented in red. The best value therefore which should be used in the model is marked in green $c_{f}=.6$. We note also for comparison that the value for $c_{f}$ obtained from classic calibration methods would have been 2 . This difference is the gain due to information quantification.

proposed here can easily be computed [40] and is found to be $c_{f}=2$. This difference between the information based parameter value $c_{f}=.6$ and its value computed based on classical approaches $c_{f}=2$ is the gain due to information quantification. We display below in Section 6 examples using this information quantification parametrization approach with data from real traffic.

Confidence Intervals for (11). Confidence intervals for the estimator $\hat{p}$ can also be derived from the pFIM. The asymptotic normality implies confidence intervals for the minimizer in (11), which is nothing but the Maximum Likelihood Estimator (see [18]). We sample the time series of consecutive pairs of samples $\sigma_{i}$ and $\sigma_{i+1}$ in the pFIM estimator past their decorrelation time. The central limit theorem therefore applies and gives the following asymptotic normality relationship $\hat{p} \rightarrow p$ almost surely with

$$
N^{1 / 2}\left(\hat{p}-p^{*}\right) \rightarrow N\left(0, F^{-1}\left(p^{*}\right)\right.
$$

where the variance is determined by the pFIM, $F\left(p^{*}\right)$ which here is given by its asymptotic $F_{n}(\hat{p})$ representation in equation (10). Therefore based on the pFIM in equation (10) we can also obtain rigorous error bars for the "best fit" minimization $p^{*}$, in (11). See also [18] for a discussion on general stochastic processes.

\section{Model optimization for US-101 in LA. Rush-hour traf- fic}

In this section we provide comparisons of the proposed RER and pFIM parametrization methodology with data from a real highway. Once again the underlying traffic flow model used in this example is from [40]. We have no indication of any equilibrium for that section of the highway and we do not try to find one. This is in fact one of the important features of the parametrization method we propose that, unlike similar methodologies, equilibrium is not necessary in order to perform the parametrization of the model parameters. 
We obtained a total of 45 minutes of highly resolved rush-hour video data from the Federal Highway Administration (FHWA) funded, NGSIM project. This video data provided detailed vehicular information from 7:50am until 8:35am for US-101 in Los Angeles California. A 15 minute interval of that data identifying a number of important traffic indicators as chosen by NGSIM is provided in red in Figures 5 and 6 .
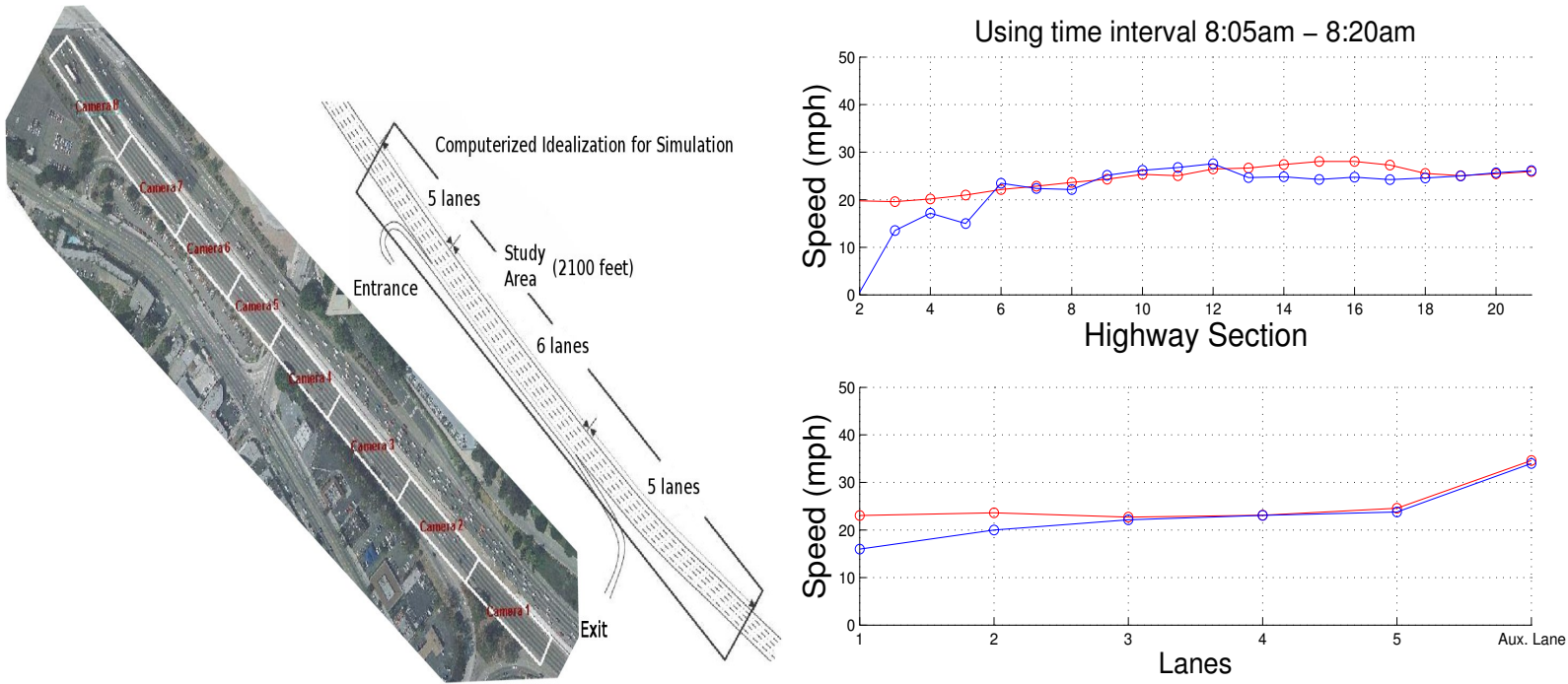

Figure 5: Geometry and comparisons. Left: highway US-101. Data provided from FHWA, NGSIM project [9]. Right. real vs simulation speeds over roadway sections (top) and lanes (bottom).

Using the microscopic model from [40] we simulate the evolution for every single vehicle in that highway and present it in blue in that same figure. The simulation is undertaken under the following geometry and boundary conditions: a) the exact same highway geometry in terms of matching number of lanes, entrances and exits and b) the exact same boundary conditions. In that respect therefore the simulation uses the real data only in terms of receiving the location and time that an actual vehicles entered the roadway. Once that vehicle is in the roadway then it's interactions and subsequent motions are simulated from the microscopic stochastic model from [40] until that vehicles exits the highway as indicated by the traffic flow model. Overall 1945 vehicles entered the roadway during that 15 minute period.

Actual data which relates to subsequent locations or velocities of those vehicles within the highway is not used at all during the simulation. This type of data on the other hand is used in the final comparisons provided in red in Figures 5 and 6 in order to illustrate the feasibility of our information theoretic approach towards automatically parameterizing our model in order to simulate an actual traffic scenario.

The overall strategy therefore suggested by the tools developed above is as follows:

- We begin with the Monte Carlo simulation which generates all the rates $r_{j}$ for the vehicle evolution and interactions based on some underlying traffic flow model.

- Model reduction. We perform sensitivity analysis of the traffic flow model parameters and eliminate those parameters which according to pFIM are not so important.

- Compute RER and update. We explore the (remaining) parameter space using RER in order to identify the most important model parameters based on current traffic information. 
Using time interval 8:05am - 8:20am
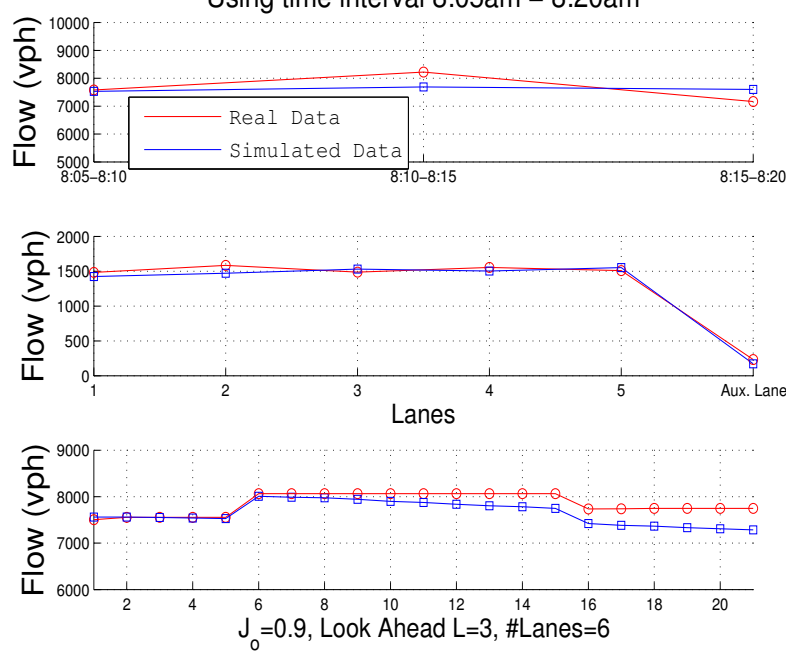
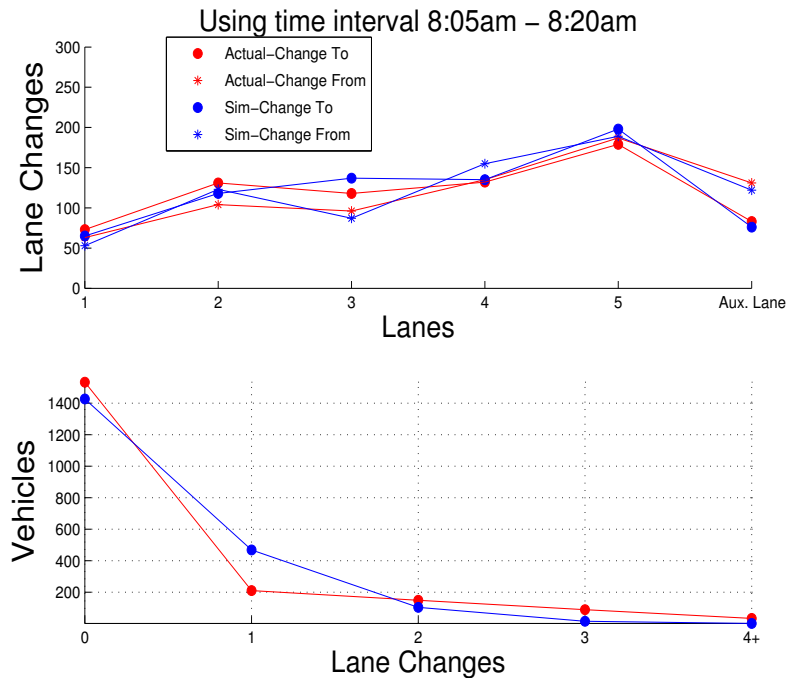

Figure 6: Comparisons between real data (red) and simulated model prediction (blue) for a 15 minute period in Highway US-101 starting at 8:05am. pFIM was first applied to reduce the model parameters. Data provided by FHWA [9]. Subsequently we update the remaining model parameters in order of importance using RER as shown in Section 5. Information used for this simulation is limited to just a) highway geometry b) location and times of vehicles entering this multi-lane highway. Real data was only used for the final comparisons. Left: real vs simulated flow over times (top) and lanes or sections (bottom). Right: lane changes comparisons between predictions and reality.

- Update all or only the important model parameters based on RER as seen in Figure 2. Choose to a) update value of the parameter by amount of perturbation if it improves RER or b) perform optimization as indicated on the next step and Section (5).

- Optional step: optimization. Compute the optimal value of the most important model parameters by minimizing the information loss as in Section 5. Always check that the optimum is within range of allowed values for that parameter.

The simulation ensues and the pFIM-RER procedure is repeated at set instances or as deemed appropriate for model adjustment and improvement. The number of times this procedure is repeated may depend on many factors such as for instance on possibly known changing traffic conditions or simply a need for higher efficiency in the model.

We provide the range that some of the model parameters span during the simulations here: $1 \leq c_{0} \leq 4.5, .1 \leq c_{f} \leq 4,1 \leq J \leq 9, .1 \leq c_{r} \leq 2.2$ and $.1 \leq c_{l} \leq 2$. In all simulations we used a look-ahead $L=3$. We also note that while the full range of those parameters is provided below the specific values of the parameters are adjusting continuously back and forth during the simulation since the traffic dynamics change over time as well as locally over space.

Overall we observe mainly satisfactory agreement of the traffic flow model simulations against actual traffic evolution over flow and velocities as presented in Figures 5 and 6. There are clearly some cases where there is room for improvement however when comparing real data against simulations. We discuss further these cases in the next section.

\subsection{Comparisons with other methods}

We now provide comparisons of the information theoretic parameterization approach proposed here against other methods. In that respect we consider two different parameterization 
and simulation packages: VISSIM and FREEVAL

VISSIM is a well-known, widely used and tested commercial software. The traffic model behind the software is the microscopic car-following model by Wiedemann [51]. The VISSIM software requires traffic data for model calibration and can be used to subsequently simulate the traffic dynamics. VISSIM furthermore includes advanced traffic features such as lookahead type of interactions which produce more realistic vehicle behavior. In other words the traffic models used within VISSIM as well as our own information-based parameterization have similar characteristics. VISSIM on the other hand can not produce the values of the model parameters in real time but instead must use traffic data in advance in order to calibrate the model for subsequent use.

FREEVAL stands for FREeway EVALuation and is an analytical tool which is suggested by the Highway Capacity Manual in order to generate density and speed predictions on a freeway segment. It incorporates procedures outlined within the Highway Capacity Manual and contains cell-transmission model based algorithms to be used in the case of oversaturated freeways. FREEVAL is able to track queue accumulation and dissipation over many segments and several time periods.

We compared both of these methods on the NGSIM data for US-101. Unfortunately the speed and density predictions from FREEVAL were very inaccurate. That may be attributed to a shortcoming of this method requiring that data inputs for the first and last time intervals to be related to uncongested regions. As a result we do not present the results from FREEVAL in our comparisons below. For further comments about FREEVAL and results specifically related to the NGSIM data we refer to page 69-75 in [16] instead. In contrast however VISSIM produced very accurate parameterizations for the underlying Wiedemann car-following model and respective speed-flow predictions which were much closer to reality. We discuss and compare these findings below.

\begin{tabular}{llll}
\multicolumn{4}{c}{ Errors in vehicle flows for each time interval } \\
\hline Model Used & $7: 50 \mathrm{am}-8: 05 \mathrm{am}$ & $8: 05 \mathrm{am}-8: 20 \mathrm{am}$ & $8: 20 \mathrm{am}-8: 35 \mathrm{am}$ \\
\hline Information Based Errors & 135.8 & 157.2 & 165.9 \\
\hline VISSIM Errors & 189.2 & 166.5 & 189.6 \\
\hline
\end{tabular}

Table 2: Error comparisons from flows for each time interval produced by either VISSIM or our method. We measured the difference between actual and predicted flows for each lane and subsequently report the error as the square root of the sum of the squared residuals. We present these comparisons for each of the three time intervals of data provided from the FHWA NGSIM project. FREEVAL errors are too high and are not presented.

We present in Table 2 the errors from using either VISSIM or our method to predict vehicle flows over each of the three 15-minute intervals provided by the NGSIM project for highway US-101 in Los Angeles. Specifically we measured the difference between actual and predicted flows over each lane and presented the errors as the square root of the sum of squares of those residuals. We present these results for each of the three 15-minute intervals available. Similar results of flow comparisons between those produced from the commercial software VISSIM and the actual NGSIM data are also found in [16]. As can be seen in Table 2 our method has consistently produced more accurate flows over all of the three time intervals available.

Lane changing information was also provided in publication [9] for the NGSIM data from FHWA. We therefore also present lane changing comparisons between the simulations produced by VISSIM and our information based-parameterization simulations in Figure 7. We include also in that figure the actual lane changing NGSIM data in red. The VISSIM lane changing data was computed from information in Table 9 on page 42 of [16]. We also computed the errors between actual and estimated lane changes as the sum of the absolute value of the residuals for the time interval 7:50 am to 8:05 am and present these results 

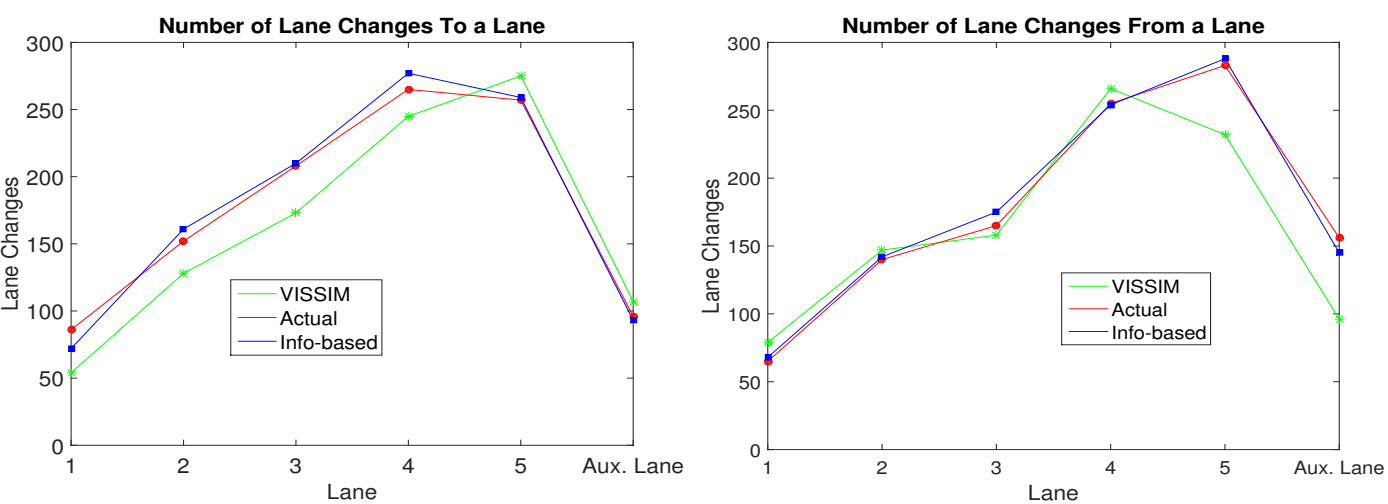

Figure 7: Number of times vehicles changed lanes to (left) and from (right) a specific highway lane. Comparisons from simulations between the VISSIM (in green) and information-based (in blue) parameterizations in contrast to the actual such number of lane changes (in red) for the time interval 7:50-8:05. Results for the time interval 8:05-8:20 and the information based parameterization are provided in the upper right corner of Figure 6.

below. In that respect the error between actual and VISSIM estimated lane changes is 140 whereas the equivalent error for our method and actual lane change is 42 . These differences can be seen in the left part of Figure 7. Similarly the error for the right part of Figure 7 between real and VISSIM estimated lane changes is 150 whereas for the information based method it is 32. Errors of similar scale between VISSIM and our approach were observed for the next time interval 8:05- 8:20. The predictions corresponding to VISSIM for the NGSIM data are further corroborated with those within [14]. In all of the cases we tested against VISSIM and FREEVAL we observe improved model parameterization and reduced errors for the information based approach we propose in this article.

\section{Conclusions and discussion}

The proposed methodology put forth in this article is substantially different from other broadly related, data-assimilation methods. The method works in path-space by analyzing information from perturbations of vehicle trajectories in order to identify optimal parameters for the underlying traffic flow model. In that respect therefore it is unique among all other such methods within the area of traffic parametrization. Furthermore the approach carries sufficient level of generality in order to be applicable to a wide class of traffic models with $\mathrm{CA}$, stochastic, KMC or Langevin dynamics. The main requirement is that some type of rule or rate/probability exists which stipulates how vehicles interact and move within the roadway. The tools presented in Section 3 and 4 for RER and pFIM can then be applied to improve and even optimize the model parameters.

This is a dynamic parametrization approach. It is therefore not surprising that this method is more computationally intensive when compared to typical traffic parametrization methods in the literature. In most traffic flow models the parameters are only initially calibrated and thereafter retained through the simulation. More advanced methods and models are able to provide predefined tables for model parameters which depend on vehicle densities. On the other hand traffic at high concentrations is a known chaotic system and in that respect no table of parameter values could possibly suffice in producing the correct parameters. This is why a dynamic approach as proposed here can be interesting in terms of providing model parameters under evolving traffic conditions. The overall computational cost for the results presented here is minimal and is performed in real time when simulating rush hour traffic in a single roadway such as highway US 101 as presented above. Performing 
equivalent computations for a complete network however is a matter for future research and parallelization of the procedure is possible and seems to be a good alternative.

The method is an inverse dynamic Monte Carlo in contrast to the inverse Monte Carlo methods typically associated to equilibrium systems. In that respect therefore the method regularly adjusts model parameters depending on prevailing traffic conditions. The adjustment of model parameters is currently performed at regular time intervals. Parameter optimization in itself as shown in Section 5 is clearly possible but not necessary. One strategy for instance is to keep updating and improving the model parameters based on the changing traffic conditions at regular time intervals. Further research will be undertaken in order to make the process more streamlined by identifying indicators after which the parametrization updating or even optimization should be performed. One such indicator for example would be to always keep track of the pFIM for significant changes. Since computation of the pFIM does not involve any substantial computational overhead that would be a cheap indicator in order to compute RER and optimize our model parameters.

The computations were performed by applying a kinetic Monte Carlo method, [5]. This is one of the most efficient, [46, 47], methods for simulating stochastic microscopic processes. Equivalent computations however would be required in the case of a Cellular Automaton model. In that respect, regardless of numerical scheme used to curry out the microscopic simulations, there is no computational overhead when it comes to computing the pFIM (10). The reason is that the pFIM uses the rates $r_{j}\left(x_{t} ; p\right)$ in order to extract information regarding model parameters. These rates however are readily available from the simulation itself. Furthermore the pFIM is gradient-free and only depends on the analytic derivatives of the propensity functions (2) with respect to the parameter vector $p$. These derivatives are explicitly computed in advance for the matrix of the pFIM and evaluated for specific $p$ as needed by the method. Thus indeed this is a gradient-free approach in contrast to other alternatives in the literature.

The RER on the other hand requires further computations to be performed due to the sensitivity analysis under specific perturbations $r_{j}\left(x_{t} ; p+\epsilon_{*}\right)$. The computed RER can be mathematically proven to be asymptotically constant, [31]. We do not need therefore to compute the complete timeseries in order to ascertain whether to update a given parameter or not. The perturbation analysis reveals, almost from the outset (see Figure 2), whether the provided perturbation should be accepted or not for that parameter. In that respect therefore these computations are not as costly given the strategy we outlined above: local timeseries computations which occur on set time instances or when there are anticipated changes in the roadway. Note that the synthetic data used to produce the results in Figures 2 and 3 was not the same and that is the reason for the different findings from this analysis.

Knowing the structure of the pFIM can help reduce the model. The pFIM signifies the important parameters which must be kept and later perturbed via RER with the vector $\epsilon_{*}$. Such an example was presented, using synthetic data, in Figure 3 where according to pFIM we should reduce the model by eliminating parameters $c_{f}, c_{r}$ and $c_{l}$. Overall therefore the computations are fast and result in improving model performance as well as speedup due to the model reduction as indicated by pFIM. The model parameters are updated as the simulation is taking place automatically providing a better fit under the available dynamic traffic information. The pFIM sensitivity analysis may allow us to consider much more complex traffic models with multiple mechanisms and features, and correspondingly many adjustable parameters that need to be fit to data. e.g. traffic lights, entrances, exits, multiple lanes/vehicle types, etc. The pFIM allows us to treat the sensitivity analysis of systems with many parameters since it is a gradient free method, e.g. [31]. In the end, the pFIM and RER analysis together produce a more efficient model adjusting to evolving dynamics than using the full original, static, traffic model.

Overall the traffic flow model simulations presented above are, for the most part, in agreement when compared against actual data. The agreement is not complete however in all cases of flow, velocity or lane changes as displayed in Figures 5 and 6 . That is perhaps 
not surprising considering that the actual road we model has 6 lanes with entrances and exits (see Figure 5, left). The significance of that is that a driver could conceivably perform moves which are not optimal in terms of their surrounding traffic. One such example for instance is when a vehicle needs to exit the highway and must therefore enter one or more lanes regardless of how many other vehicles may already occupy those lanes. Such and many other similar scenarios which make sense from the driver's perspective might be avoided by the simulations trying to investigate the parameter space in order to minimize information loss. In that respect therefore the example we provided for highway US-101 above is more of a toughest case scenario instead of a usual test case. This is a research direction, to be undertaken in the future, where we should explore further the dynamics and understand if it is possible to influence RER to anticipate such demands. One way to do that would be to also include information in terms of the expected number of drivers exiting the roadway.

The proposed tools do not by themselves resolve the many issues discussed above. As a result the proposed methodology can be useful in conjunction with other sensitivity analysis tools such as those in [3] or [18] and [34] in order to further help eliminate unimportant parameters and/or differentiate among the most sensitive parameters. Furthermore the underlying traffic flow model upon which this optimization is performed can be based on either microscopic or macroscopic type models such as for instance the model by $[34,48]$ or [52]. Although this is a task to be resolved in the future.

In terms of future work it would be interesting to extend our parameterization approach to macroscopic models. We can consider for instance connections with the anisotropic macroscopic model in [53] as well as the multi-class model in [54]. Specifically the microscopic traffic model in [40] has been shown, in the asymptotic limit of long-range interactions $L \rightarrow \infty$, to produce the Lighthill-Whitham model at the zeroth-level expansion. Higher order traffic equations are also provided at different levels of the asymptotic expansion, such as Eq. 4.16 in [40]. There exist therefore a natural connection between the microscopic model here and the macroscopic model in [40]. As a result there is a one to one correspondence between the respective microscopic and macroscopic model parameters. Due to the similarities of [40] with either [54] or [53] it would be possible to compare the results from our parameterization methodology here with either of the models above. Specifically after appropriate rescaling of the parameters we could obtain the link between the current parameter $c_{0}$ and the parameter $1 / \beta$ in the higher-order model equation (9) in [53]. It would therefore be possible to study the equilibrium and non-equilibrium behavior between these two models based on their microscopic and macroscopic formulations respectively.

\section{Acknowledgements}

The work of A.S. was supported in part by the Swedish innovation agency, Vinnova, proj. ID: VLU12-674c. The work of M.A.K. was supported in part by the Office of Advanced Scientific Computing Research, U.S. Department of Energy under Contract No. DE-SC0010723.

\section{Appendix A. Relative entropy and information quantifi- cation}

We outline here how relative entropy is defined and its uses through statistical mechanics and information theory applications. We refer to [30] and [18] for all the details. The relative entropy is a semi-metric and defined via,

$$
\mathcal{R}\left(Q_{[0, T]}^{p} \mid Q_{[0, T]}^{q}\right)=\int \log \left(\frac{d Q_{[0, T]}^{p}}{d Q_{[0, T]}^{q}}\right) d Q_{[0, T]}^{p},
$$


where $Q_{[0, T]}^{p}$ and $Q_{[0, T]}^{q}$ are two path-space probability measures for continuous time Markov Chains over a given time interval $[0, T]$. Using this quantity we can therefore ascertain "distance" or the difference between these two measures. Furthermore from an information theory perspective, as presented in [6], the relative entropy measures loss/change of information. To simplify (12) we apply Girsanov's formula and obtain an explicit expression for the Radon-Nikodym derivative appearing in (12). The result is that the relative entropy is split into two parts,

$$
\mathcal{R}\left(Q_{[0, T]}^{p} \mid Q_{[0, T]}^{q}\right)=\operatorname{TH}\left(Q_{[0, T]}^{p} \mid Q_{[0, T]}^{q}\right)+\mathcal{R}\left(\mu^{p} \mid \mu^{q}\right) .
$$

The part with $\mathcal{R}\left(\mu^{p} \mid \mu^{q}\right)$ scales as $\mathcal{O}(1)$ and is capturing the stationary dynamics with respect to the stationary probability $\mu$. The other part, $\mathcal{H}$, is the relative entropy rate, RER,

$$
\mathcal{H}\left(Q_{[0, T]}^{p} \mid Q_{[0, T]}^{q}\right)=\mathbf{E}_{\mu}\left[\sum_{\sigma^{\prime}} \lambda^{p}(\sigma) \nu^{p}\left(\sigma, \sigma^{\prime}\right) \log \frac{\lambda^{p}(\sigma) \nu^{p}\left(\sigma, \sigma^{\prime}\right)}{\lambda^{q}(\sigma) \nu^{q}\left(\sigma, \sigma^{\prime}\right)}-\lambda^{p}(\sigma)+\lambda^{q}(\sigma)\right] .
$$

The relative entropy rate scales as $\mathcal{O}(T)$ and is tracking the transient states. Here $\lambda^{p}(\sigma)=$ $\sum_{\sigma^{\prime}} r^{p}\left(\sigma, \sigma^{\prime}\right)$ with $r^{p}\left(\sigma, \sigma^{\prime}\right)$ defined in $(2)$ and $\nu^{p}\left(\sigma, \sigma^{\prime}\right)=r^{p}\left(\sigma, \sigma^{\prime}\right) / \lambda^{p}(\sigma)$. When replacing $Q_{[0, T]}^{q}$ with $Q_{[0, T]}^{p+\epsilon}$ the quantity $\mathcal{H}$ above is viewed as a way to quantify parameter sensitivity.

Assuming smoothness in the parameter vector $p$ we expand (12) as in [6] and obtain $\mathcal{R}\left(Q_{[0, T]}^{p} \mid Q_{[0, T]}^{p+\epsilon}\right)=\frac{1}{2} \epsilon^{T} F_{\mathcal{R}}\left(Q_{[0, T]}^{p}\right) \epsilon+\mathcal{O}\left(\left|\epsilon^{3}\right|\right)$ where the Fisher information matrix is defined as the Hessian of the relative entropy $F_{\mathcal{R}}\left(Q_{[0, T]}^{p}\right)=\nabla_{\epsilon}^{2} \mathcal{R}\left(Q_{[0, T]}^{p} \mid Q_{[0, T]}^{p+\epsilon}\right)$. Combining this equation with (13) and (14) gives the pFIM,

$$
F_{\mathcal{R}}\left(Q_{[0, T]}^{p}\right)=\mathbf{E}_{\mu}\left[\sum_{\sigma^{\prime}} r^{p}\left(\sigma, \sigma^{\prime}\right) \nabla_{p} \log r^{p}\left(\sigma, \sigma^{\prime}\right) \nabla_{p} \log r^{p}\left(\sigma, \sigma^{\prime}\right)\right] .
$$

The estimators for RER, (9) and pFIM, (10) provided in Sections 3 and 4 respectively are direct consequences of the formulas (14) and (15) above.

For more details of these derivations and connections to information theory we refer to $[30]$ and $[6]$.

\section{Appendix B. Statistical mechanics notion of equilibrium}

We provide in this appendix a more concrete mathematical description of the concept of equilibrium from a statistical mechanics point of you. At the same time we provide simple examples of that concept in the realm of vehicular traffic. To better understand however what we mean by equilibrium we must first briefly explain and review concepts such as Markov chain and transition probability matrix between system states.

A discrete time Markov chain, such as the one used in this article, is a random process that undergoes transitions from one state to another on a state space $\Lambda$. For our example in traffic modeling the state space $\Lambda$ therefore consists of all possible configurations that the traffic fleet can acquire on that highway. Furthermore the Markov chain is the random process $\left\{\sigma_{t}\right\}_{t \geq 0}$ which we presented in Section 2 in order to simulate how vehicles move forward and interact with their neighbors on that highway. The states within which the system moves through consist of the different configurations that the traffic stream of vehicles attains from one time instant to another.

Let us consider a system in state $x$ at time $t$ and assume that we know the probabilities that the system will move to a new state $y$ at time $t+\Delta t$. We put together these probabilities into the transition probability matrix $P_{i j}$ which describes how the system will change from one state $i$ in the state space $\Lambda$ to another state $j \in \Lambda$. The matrix $P_{i j}$ is responsible for 
the resulting dynamics of that stochastic system. We can now define the vector $\pi$ which is called the stationary or equilibrium distribution for the system if,

$$
\pi_{i}=\sum_{j \in \Lambda} \pi_{j} P_{i j}, \quad \text { where } \quad \sum_{j \in \Lambda} \pi_{j}=1 \quad \text { and } \quad 0 \leq \pi_{j} \leq 1 .
$$

Note that $\pi$ is a probability distribution function or pdf. It is possible that a given system has more than one states which satisfy the above equilibrium. In that respect the system could, under the influence of noise in the Monte Carlo Markov chain and depending on random fluctuations, visit one or many of those other equilibrium states. From the perspective of our example in traffic dynamics this is therefore equivalent to vehicles changing speeds and spacings as they visit from equilibrium state to equilibrium state. As a result a given traffic network which is already in an equilibrium state could switch to a different state with same equilibrium given sufficient noise in the system.

In essence therefore the statistical mechanics equilibrium we described here is slightly more general than Wardrop equilibrium. The major difference is that due to the Monte Carlo Markov Chain we are able to visit all possible states which belong to the probability distribution function satisfying (16). Similarly, another notable difference however is that even if a given traffic fleet has reached an equilibrium state their velocities and spacing may fluctuate depending on the noise of that traffic system.

\section{References}

[1] T. Alperovich and A. Sopasakis, Stochastic description of traffic flow, Journal of Stat. Phys. 133 (6), pp. 1083 - 1105, (2008).

[2] D. F. Anderson, An efficient finite difference method for parameter sensitivities of continuous-time Markov chains, SIAM J. Numer. Anal., 50 (5), pp. 2237 - 2258, (2012).

[3] G. Arabatzis and M. A. Katsoulakis, Goal-oriented sensitivity analysis for lattice kinetic Monte Carlo simulations, J. of Chem. Phys., 140 (12), (2014).

[4] R. Bishop, Intelligent Vehicle Technology and Trends. Artech House Publishers, (2005).

[5] A. B. Bortz, M. H. Kalos, and J. L. Lebowitz. A new algorithm for Monte Carlo simulations of Ising spin systems, J. Comput. Phys., 17:10, (1975).

[6] T. M. Cover and J. A. Thomas, Elements of Information Theory. Wiley Series in Telecommunications, (1991).

[7] N. Dundon and A. Sopasakis, Stochastic modeling and simulation of multi-lane traffic, in ISTTT 17, pp. 661-689, (2007).

[8] A. F. Emery and A. V. Nenarokomov, Optimal experiment design, Meas. Sci. Technol., (9), pp. 864-876, (1998).

[9] Federal Highway Administration, Data Analysis Report, http://ngsim-community . org/US-101/US-101-Data-Analysis-Report.zip, accessed:2015-07-01.

[10] S. Fan and D. B. Work, A heterogeneous multiclass traffic flow model with creeping, SIAM J. Appl. Math., 75 (2), pp. 813-835, (2015).

[11] P. W. Glynn, Likelihood ratio gradient estimation for stochastic systems, Commun. ACM, 33 (10), pp. 75-84, (1990). 
12] V. Harmandaris, E. Kalligiannaki, M. A. Katsoulakis and P. Plecháč, Path-space variational inference for non-equilibrium coarse-grained systems, ArXiv e-prints 1508.00289, Provided by the SAO/NASA Astrophysics Data System, (2015).

[13] D. Helbing, A. Hennecke, V. Shvetsov, and M. Treiber, Micro- and macrosimulation of freeway traffic, Math. Comput. Modelling, 35, pp. 517-547, (2002).

[14] W.E. Hughes, R. Jagannatan, M. Qi and N. Ghanta, Safety impacts of new traffic management techniques, by Vanasse Hangen Brustlin Inc. for the Office of the Secretary of Transportation, Nov. 30, 2011.

[15] H. Jeffreys, Theory of Probability. Oxford, Oxford University Press, (1948).

[16] D. Jolovic, Use of microsimulation to access HCM 2010 methodology for oversaturated freeway segments, Florida Atlantic University Thesis, May 2012.

[17] J. Kaipioa and E. Somersalob, Statistical inverse problems: Discretization, model reduction and inverse crimes, Journal of Computational and Applied Mathematics, 198 (2), pp. 493-504, (2007).

[18] M. A. Katsoulakis and P. Plecháč, Information-theoretic tools for parametrized coarsegraining of non-equilibrium extended systems, J. Chem. Phys. 139, 074115, (2013).

[19] T. Kim and H. M. Zhang, A stochastic wave propagation model, Transp. Res. Part B, 7-8 (42), pp. 619-634, (2008).

[20] M. Komorowski, M. J. Costa, D. A. Rand and M. P. H. Stumpf, Sensitivity robustness and identifiability in stochastic chemical kinetics, in Proc. Natl. Acad. Sci. USA, 108, pp. 8645-8650, (2011).

[21] M. Komorowski, J. Zurauskiene and M. Stumpf, StockSens-Matlab package for sensitivity analysis of stochastic chemical systems, Bioinformatics, (28), pp. 731-733, (2012).

[22] I. J. Leontaridis and S. A. Billings, Experimental design and identifiability for non-linear systems, Int. J. Systems, (18), pp. 189-202, (1987).

[23] T. M. Liggett, Interacting Particle Systems. Springer, (1985).

[24] H. S. Mahmassani and J. Dong, Stochastic modeling of traffic flow breakdown phenomenon: Application to predicting travel time reliability, in IEEE Transactions on Intelligent Transportation Systems, (2012).

[25] D. J. C. MacKay, Information Theory, Inference \& Learning Algorithms. New York, NY, USA, (2003).

[26] T. Morimura, T. Osogami and T. Ide, Solving inverse problem of Markov chain with partial observations, in Proc. of Neural Inform. and Processing Systems, pp. 1655-1663, (2013).

[27] K. Nagel and M. Shreckenberg, A cellular automaton model for freeway traffic, Journal de physique, I2 (12), pp. 2221-2229, (1992).

[28] M. Nakayama, A. Goyal, and P. W. Glynn, Likelihood ratio sensitivity analysis for Markovian models of highly dependable systems, Stochast. Models 10, pp. 701-717, (1994).

[29] K. Nagel, D. E. Wolf, P. Wagner and P. Simon, Two-lane traffic rules for cellular automata: a systematic approach, Physical Review E, 58 (2), pp. 1425-1437, (1998). 
[30] Y. Pantazis and M. A. Katsoulakis, A relative entropy rate method for path space sensitivity analysis of stationary complex stochastic dynamics, J. Chem. Phys., 138 (5), 054115, (2013).

[31] Y. Pantazis, M. A. Katsoulakis and D. G. Vlachos, Parametric sensitivity analysis for biochemical reaction networks based on pathwise information theory, BMC Bioinformatics, 14:311, (2013).

[32] S. Plyasunov and A. P. Arkin, Efficient stochastic sensitivity analysis of discrete event systems, J. Comput. Phys., 221, pp. 724-738, (2007).

[33] M. Rathinam, P. W. Sheppard, and M. Khammash, Efficient computation of parameter sensitivities of discrete stochastic chemical reaction networks, J. Chem. Phys., 132, 034103, pp. 1-13, (2010).

[34] C. Roncoli, M. Papageorgiou and I. Papamichail, An optimization-oriented first-order multi-lane model for motorway traffic, in Proc. of the 94th Annual Meeting of the Transp. Res. Board, Washington D.C., USA, 11-15 January 2015.

[35] P. W. Sheppard, M. Rathinam, and M. Khammash, A pathwise derivative approach to the computation of parameter sensitivities in discrete stochastic chemical systems, J. Chem. Phys., 136 (3), 034115, (2012).

[36] U. Sparmann, Spurwechselvorgänge auf Zweispurigen BAB-Richtungsfahrbahnen, Forschung Straßenbau und Straßenverkehrstechnik, Bundesminister für Verkehr, BonnBad Godesberg, (1978).

[37] A. Sopasakis, Stochastic noise approach to traffic flow modeling, Physica A, pp. 741$754,(2004)$.

[38] A. Sopasakis, Lattice free stochastic dynamics, Comm. Comp. Phys., 12 (3), pp. 691$702,(2012)$.

[39] A. Sopasakis, Traffic updating mechanisms for stochastic lattice-free dynamics, Procedia - Social and Behavioral Sciences, 80 (7), pp. 837-845, (2013).

[40] A. Sopasakis and M. A. Katsoulakis, Stochastic modeling and simulation of traffic flow: ASEP with Arrhenius look-ahead dynamics, SIAM J.Appl. Math., (66), pp. 921-944, (2006).

[41] H. Spohn, Large Scale Dynamics of Interacting Particles. Springer-Verlag, (1991).

[42] O. Tossavainen and D. Work, Markov chain Monte Carlo based inverse modeling of traffic flows using GPS data, Networks and Heterogeneous Media, 8 (3), pp. 803-824, (2013).

[43] M. Treiber, A. Hennecke and D. Helbing, Congested traffic states in empirical observations and microscopic simulations, Physical Review E, 62 (2), pp. 1805, (2000).

[44] M. Treiber and A Kerstin, Traffic Flow Dynamics: Data Models and Simulation. Springer, (2013).

[45] A. Schadschneider, Traffic flow: a statistical physics point of view, Physica A, 312:153, (2002).

[46] T. P. Schulze, Efficient kinetic Monte Carlo simulation, Journal of Computational Physics, 227 (4), pp. 2455-2462, (2008). 
[47] A. Slepoy, A. P. Thompson, and S. J. Plimpton, A constant-time kinetic Monte Carlo algorithm for simulation of large biochemical reaction networks, Journal of Chemical Physics, 128 (20), 205101, (2008).

[48] Y. Wang, M. Papageorgiou and A. Messmer, RENAISSANCE - A unified macroscopic model-based approach to real-time freeway network traffic surveillance, Transp. Res. Part C: Emerging Technologies, (14), pp. 190-212, (2006).

[49] J. G. Wardrop, Some theoretical aspects of road traffic research, in Proceedings of the Institut. of Civil Engineers, Part II, pp. 325-378, (1952).

[50] L. Wasserman, All of Statistics: A Concise Course in Statistical Inference. Springer, (2004).

[51] R. Wiedemann, Simulation des Strassenverkehrsflusses, Schriftenreihe des Instituts für Verkehrswesen Heft 8, Universität Karlsruhe, (1974).

[52] M. Yildirimoglu and N. Gerolimitis, Approximating dynamic equilibrium conditions with macroscopic fundamental diagrams, Transp. Res. part b, (70), pp. 186-200, (2014).

[53] P. Zhang, S. C. Wong and S. Q. Dai, A conserved higher-order anisotropic traffic flow model: description of equilibrium and non-equilibrium flows, Transp. Res. Part b, (43), pp. 562-574, (2009).

[54] P Zhang, R. X. Liu, S. C. Wong and S. Q. Dai, Hyperbolicity and kinematic waves of a class of multi-population partial differential equations, Euro. Jnl of Applied Mathematics, (17), pp. 171-200, (2006). 